\title{
EPIC STAR: a reliable and efficient approach for phonon- and impurity-limited charge transport calculations
}

\author{
Tianqi Deng $\mathbb{D}^{1}$, Gang Wu $\mathbb{D}^{1 凶}$, Michael B. Sullivan ${ }^{1}{ }^{1}$, Zicong Marvin Wong ${ }^{1}$, Kedar Hippalgaonkar ${ }^{2,3}$, Jian-Sheng Wang ${ }^{4}$ and \\ Shuo-Wang Yang ${ }^{\text {网 }}$
}

A computationally efficient first-principles approach to predict intrinsic semiconductor charge transport properties is proposed. By using a generalized Eliashberg function for short-range electron-phonon scattering and analytical expressions for long-range electron-phonon and electron-impurity scattering, fast and reliable prediction of carrier mobility and electronic thermoelectric properties is realized without empirical parameters. This method, which is christened "Energy-dependent Phonon- and Impuritylimited Carrier Scattering Time AppRoximation (EPIC STAR)" approach, is validated by comparing with experimental measurements and other theoretical approaches for several representative semiconductors, from which quantitative agreement for both polar and non-polar, isotropic and anisotropic materials is achieved. The efficiency and robustness of this approach facilitate automated and unsupervised predictions, allowing high-throughput screening and materials discovery of semiconductor materials for conducting, thermoelectric, and other electronic applications.

npj Computational Materials (2020)6:46; https://doi.org/10.1038/s41524-020-0316-7

\section{INTRODUCTION}

Computational screening of electronic materials, empowered by new theories, computational tools and high computing power, is an efficient and economical alternative of purely experimental exploration. For instance, these tools have assisted the discovery of many new high performance thermoelectric (TE) candidates, such as $\mathrm{TmAgTe}_{2}{ }^{1}, \mathrm{NbFeSb}^{2}, \mathrm{NbCoSn}^{3}$, and $\mathrm{Cdln}_{2} \mathrm{Te}_{4}{ }^{4}$, to name a few. High mobility oxides ${ }^{5}$ have also been explored to uncover new materials as transparent conductors with potential application in display, light-emitting diodes, and solar cells. Some computationally identified candidates, such as $\mathrm{Ba}_{2} \mathrm{BiTaO}_{6}{ }^{6}$ and TalrGe ${ }^{7}$, have been experimentally verified. Many of the previous works are based on the prediction of carrier mobility $\mu$, electrical conductivity $\sigma$, and Seebeck coefficient $S$. Although the state-ofthe-art tools and theories based on Boltzmann transport equation (BTE) are able to accurately predict both electron ${ }^{8,9}$ and phonon $^{10,11}$ transport properties, the high complexity and computational cost make it a challenge for high-throughput screening of possible materials. To overcome this, various approximations have been established to make the calculations more tractable.

To determine $\mu, \sigma$, and $S$, BTE solution within scattering time approximation (STA) is commonly adopted ${ }^{12}$ which yields good agreement with fully numerical solution of the equation ${ }^{13}$. However, to determine the phonon-limited intrinsic transport properties, very dense sampling of both electron and phonon Brillouin zones are required for the integration. $10^{5}-10^{6} \mathbf{k}$ and $\mathbf{q}$ points are needed for a material as simple as $\mathrm{Si}^{9,13,14}$ and even more for polar semiconductors such as $\mathrm{GaAs}^{14-16}$. This is computationally complex and expensive even with the use of linear $^{13}$ or Wannier ${ }^{8,17}$ interpolation techniques. Therefore, constant scattering time approximation (CSTA) is commonly employed in tools such as BoltzTraP ${ }^{18,19}$ and BoltzWann ${ }^{20}$ where only electronic structure calculation from density functional theory $(\mathrm{DFT})^{21}$ is needed, and it indeed provides a lot of insights into the role of the electron band structure. For example, high band degeneracy was found to be related to high $z T$, whereas band convergence, which increases such degeneracy, has become an effective method for enhancing TE performance ${ }^{1,22,23}$. Quantitative descriptors calculated in CSTA such as Fermi surface complexity factor ${ }^{24}$ and electronic fitness function ${ }^{25}$ have also been proposed as screening criteria for TE materials.

While CSTA could predict the Seebeck coefficient, which is independent of scattering time $\tau$ in CSTA, the carrier mobility and electrical conductivity inevitably vary as the magnitude of $\tau$ differs from one system to another. It is also found that when $\tau$ depends on the electron energy $E$, this dependency would also affect the value of $S^{26-29}$. As such, this hinders the predictive power of CSTA, implying the need for evaluation of $\tau$ from first-principles by including important scattering mechanisms such as electron-phonon interaction and charged impurity scattering. Deformation potential theory (DPT), pioneered by Bardeen and Shockley ${ }^{30}$, describes the scattering from long-wavelength acoustic phonons, which is an important scattering mechanism in semiconductors. It has been widely used in predicting TE properties of various materials ${ }^{31-36}$ and recently in highthroughput search for new TE compounds ${ }^{4}$. The electron-phonon averaged (EPA) approximation ${ }^{37}$ takes into account all electron-phonon interactions and uses the average coupling to evaluate $\tau$. It achieves quantitative agreement with full and accurate calculations using electron-phonon Wannier (EPW) method $^{8}$ for some half-Heusler compounds in the heavily doped region. However, the absence of polar optical phonon (POP) scattering impairs the accuracy of $\tau$ predictions, whereby the scattering time and performance may be overestimated for certain systems. For example, in lightly doped half-Heusler compounds,

\footnotetext{
${ }^{1}$ Institute of High Performance Computing, Agency for Science, Technology and Research, 1 Fusionopolis Way, \#16-16 Connexis, Singapore 138632, Singapore. ${ }^{2}$ School of Materials Science and Engineering, Nanyang Technological University, 50 Nanyang Avenue, Singapore 639798, Singapore. ${ }^{3}$ Institute of Materials Research and Engineering, Agency for Science, Technology and Research, 2 Fusionopolis Way, \#08-03 Innovis, Singapore 138634, Singapore. ${ }^{4}$ Department of Physics, Faculty of Science, National University of Singapore, 2 Science Drive 3, Singapore 117551, Singapore. ${ }^{凶}$ email: wug@ihpc.a-star.edu.sg; yangsw@ihpc.a-star.edu.sg
} 
optical phonons dominate the scattering of charge carriers near band edges as a consequence of weak electron-acoustic-phonon coupling ${ }^{38}$. Therefore the intrinsic carrier mobility may be overestimated. In fact, we will show that EPA predicts an intrinsic hole mobility of $149 \mathrm{~cm}^{2} \mathrm{~V}^{-1} \mathrm{~s}^{-1}$ for NbFeSb which is twice the value from EPW calculation ${ }^{38}\left(77 \mathrm{~cm}^{2} \mathrm{~V}^{-1} \mathrm{~s}^{-1}\right)$. Direct averaging of electron-phonon matrix elements and frequencies may not be appropriate for acoustic phonons and POP due to large variations of their magnitudes in Brillouin zone ${ }^{39}$. Moreover, using one single frequency for a whole phonon branch is not accurate especially for acoustic phonons, where long wavelength electron-phonon scattering is almost elastic. Therefore, a more general approximation is needed to incorporate the appropriate treatment for all phonon branches. The short-range electron-phonon interaction in metals, which is important for the prediction of conventional superconductor and metal resistivity ${ }^{40}$, was described by Eliashberg electron-phonon spectral function $a^{2} F(\omega)$ which was used in Allen-Dynes formula for transition temperature ${ }^{41,42}$ and Ziman's resistivity formula ${ }^{12,43}$. Its dependence on phonon frequency $\omega$ could reflect some details that are missing in DPT and EPA methods, and first-principles calculation of $a^{2} F(\omega)$ has been demonstrated for systems like $\mathrm{Nb}^{44,45}, \mathrm{Al}^{13}, \mathrm{~Pb}^{8,45}$, and B-doped diamond ${ }^{46}$. Inspired by these works, the extension of Eliashberg function to non-metallic systems could potentially be a feasible approach to realize fast and accurate prediction of short-range electron-phonon interactions and charge transport properties. And the long-range electron-phonon scattering can be treated analytically due to their diverging behavior for small momentum phonons ${ }^{47}$. The charged impurity scattering could also be treated in a similar way as a Coulomb potential from point charges.

In this work, we propose a new first-principles approach for "Energy-dependent Phonon- and Impurity-limited Carrier Scattering Time AppRoximation (EPIC STAR)" from density functional perturbation theory (DFPT) calculations, which overcomes the above-mentioned difficulties with reliable prediction for electron scattering from all phonon branches. The EPIC STAR calculation after obtaining DFPT phonon and electron-phonon information is very fast and computational cost is only limited by DFPT itself. This new approach treats the short- and long-range scattering mechanisms separately to reproduce their different behavior. Specifically, it employs analytical expressions to treat the longrange POP scattering and impurity scattering, using ab initio information including Born effective charges and dielectric tensor; and the remaining short-range electron-phonon interactions are described numerically using a generalized Eliashberg function, $a^{2} F(E, \omega)$ from first-principles calculations which depends on both electron energy $E$ and phonon frequency $\omega$. The long-range POP scattering formulism is based on recent works for first-principles treatment of Fröhlich interactions in polar materials 4748 , and impurity scattering is simulated with Brooks-Herring mode ${ }^{39,49,50}$. $a^{2} F(E, \omega)$ is a generalization of the electron-phonon spectral function where $E$-dependence and energy conservation are explicitly included. The new approach is validated for $\mathrm{Si}, \mathrm{GaAs}$, $\mathrm{Mg}_{2} \mathrm{Si}$, and $\mathrm{NbFeSb}$, which represent high mobility and high TE performance materials with different extents of polarization and band structure anisotropy. EPIC STAR calculation also reveals a new TE material, NalnSe 2 whose performance has not been studied before. The agreement with EPW prediction and experimental measurements is investigated and high predictive capability is achieved at costs lower by at least 1 order of magnitude. The computational cost is not just similar to those of phonon calculations and the EPA method, but also reliable for more general family of materials with improved accuracy. Moreover, the DFPT calculations for EPIC STAR will not be wasted as they can be directly fed into accurate calculations like EPW if necessary, while the phonon-related information can be utilized in other relative studies such as thermal, elastic and piezoelectric properties. This approach is therefore suitable for automated charge transport property calculation and high-throughput screening of electronic materials with different functionalities arising from non-equilibrium electron scattering times, such as thermoelectrics, high power electronics and photovoltaics.

\section{RESULTS AND DISCUSSIONS}

Energy-dependent scattering time approximation

The electron energy scattering time limited by electron-phonon interactions is given by ${ }^{40}$

$$
\begin{aligned}
\tau_{n \mathbf{k}}^{-1}= & \sum_{l} \tau_{l n \mathbf{k}}^{-1}=\sum_{l m} \frac{2 \pi}{\hbar} \int_{B Z}\left|g_{n m l}(\mathbf{k}, \mathbf{q})\right|^{2} \\
& {\left[\left(1-f_{m \mathbf{k}+\mathbf{q}}^{0}+n_{l \mathbf{q}}^{0}\right) \delta\left(\varepsilon_{n \mathbf{k}}-\hbar \omega_{l \mathbf{q}}-\varepsilon_{m \mathbf{k}+\mathbf{q}}\right)+\left(f_{m \mathbf{k}+\mathbf{q}}^{0}+n_{l \mathbf{q}}^{0}\right) \delta\left(\varepsilon_{n \mathbf{k}}+\hbar \omega_{l \mathbf{q}}-\varepsilon_{m \mathbf{k}+\mathbf{q}}\right)\right] \frac{d \mathbf{q}}{\Omega_{B Z}} }
\end{aligned}
$$

where $g_{n m l}(\mathbf{k}, \mathbf{q})$ is the electron-phonon coupling matrix element among electrons in state $n \mathbf{k}$, state $m \mathbf{k}+\mathbf{q}$, and phonon in branch / with momentum q. $n_{l q}^{0}$ is the Bose-Einstein distribution function of phonons with frequency $\omega_{/ \mathbf{q}}$ while $f_{m \mathbf{k}+\mathbf{q}}^{0}$ is the Fermi-Dirac distribution function of electrons with energy $\varepsilon_{m \mathbf{k}+\mathbf{q}}$. The phonon frequencies and electron-phonon coupling matrix can be obtained from density functional perturbation theory (DFPT) calculation as implemented in PHonon code in QUANTUM ESPRESSO ${ }^{51,52}$.

While direct evaluation of Eq. (1) becomes available using interpolation techniques for various materials ${ }^{14,53-55}$, it is still computationally expensive. To simplify the computation in EPIC STAR, we again reduce the complex $n \mathbf{k}$-dependent scattering rate to an energy-dependent averaged scattering rate $\tau^{-1}(E)$. This is exact in the case of an isotropic band dispersion ${ }^{39}$. For anisotropic system, it is still a reasonable approximation which will be verified in this work. In this approximation, the transport distribution function simply becomes

$$
[\boldsymbol{\Sigma}(E)]_{a \beta} \approx \overline{v_{\alpha \beta}^{2}}(E) \rho(E) \tau(E)
$$

where the density of states $\rho(E)$, averaged squared velocity tensor $v_{a \beta}^{2}$ and averaged scattering rate $\tau^{-1}$ can be calculated from band structure as ${ }^{18,37}$

$$
\begin{aligned}
& \rho(E)=\sum_{n} \int_{B Z} \delta\left(E-\varepsilon_{n \mathbf{k}}\right) \frac{d \mathbf{k}}{\Omega_{B Z}} \\
& \overline{v_{\alpha \beta}^{2}}(E)=\frac{1}{\rho(E)} \sum_{n} \int_{B Z} v_{n \mathbf{k}}^{a} v_{n \mathbf{k}}^{\beta} \delta\left(E-\varepsilon_{n \mathbf{k}}\right) \frac{d \mathbf{k}}{\Omega_{B Z}} \\
& \tau^{-1}(E)=\sum_{l} \tau_{l}^{-1}(E)=\sum_{l} \frac{1}{\rho(E)} \sum_{n} \int_{B Z} \tau_{l n \mathbf{k}}^{-1} \delta\left(E-\varepsilon_{n \mathbf{k}}\right) \frac{d \mathbf{k}}{\Omega_{B Z}}
\end{aligned}
$$

In the EPA approximation ${ }^{37}$, the energy-dependent scattering time is obtained by replacing electron-phonon matrix element squared $\left|g_{n m l}(\mathbf{k}, \mathbf{q})\right|^{2}$ with its average value at energy pair for $\varepsilon_{n \mathbf{k}}$ and $\varepsilon_{m \mathbf{k}+\mathbf{q}}$. This can be achieved by energy-bin-based averaging ${ }^{37}$ or moving least squares averaging (MLS-EPA) ${ }^{56}$. This is also well justified for non-polar optical phonon scattering and inter-valley scattering, where the matrix element varies slowly and can be approximated by the corresponding optical or intervalley deformation potential constants ${ }^{39}$. However, we noticed that such treatment may not be good enough in cases where $\left|g_{n m l}(\mathbf{k}, \mathbf{q})\right|^{2}$ varies rapidly. This is particularly important for intra-valley acoustic phonon scattering, where $\left|g_{n m l}(\mathbf{k}, \mathbf{q})\right|^{2} \propto|\mathbf{q}|$, and POP scattering where $\left|g_{n m l}(\mathbf{k}, \mathbf{q})\right|^{2} \propto|\mathbf{q}|^{-2}$. In the first case, while $\left|g_{n m l}(\mathbf{k}, \mathbf{q})\right|^{2}$ is linear for long-wavelength acoustic phonons, its Bose-Einstein distribution is not exactly $|\mathbf{q}|^{-1}$ if temperature is not very high. Therefore, separated averaging of $\left|g_{n m l}(\mathbf{k}, \mathbf{q})\right|^{2}$ and $\omega_{\text {lq }}$ may lead to different results in such case. For POP scattering, the scattering matrix diverges as $|\mathbf{q}|^{-2}$ which decays rapidly as $|\mathbf{q}|$ increases, so the average value cannot represent the scattering strength. 
In accordance with the $\mathrm{DPT}^{30}$, both the electron-acousticphonon matrix and the phonon dispersion are linear in $|\mathbf{q}|$ around $\Gamma$ point $^{38}$. Therefore, it can be expected that $\left|g_{n m l}(\mathbf{k}, \mathbf{q})\right|^{2} / \omega_{/ \mathbf{q}}$ varies slowly for long wavelength and could be a better candidate for averaging. This also holds for non-polar optical phonon scattering and inter-valley scattering as both $\left|g_{n m l}(\mathbf{k}, \mathbf{q})\right|^{2}$ and $\omega_{/ \mathbf{q}}$ vary slowly for these cases. With this in mind, we define a generalized Eliashberg function for short-range electron-phonon interaction $^{43}$

$$
\begin{aligned}
a_{l}^{2} F(E, \omega) & =\frac{\omega}{\rho(E)} \sum_{m n} \int_{B Z} \frac{d \mathbf{k}}{\Omega_{B Z}} \frac{d \mathbf{q}}{\Omega_{B Z}} \frac{\left|g_{n m l}^{S}(\mathbf{k}, \mathbf{q})\right|^{2}}{\omega_{l \mathbf{q}}} \delta\left(E-\varepsilon_{n \mathbf{k}}\right) \\
& \times \delta\left(\hbar \omega+E-\varepsilon_{m \mathbf{k}+\mathbf{q}}\right)\left[\delta\left(\omega-\omega_{l \mathbf{q}}\right)+\delta\left(\omega+\omega_{l \mathbf{q}}\right)\right]
\end{aligned}
$$

Here, the energy and momentum conservations during electron-phonon scattering are preserved through Dirac delta functions, while the positive and negative $\omega$ stand for phonon absorption and emission, respectively. The matrix $\left|g_{n m l}^{s}(\mathbf{k}, \mathbf{q})\right|^{2}$ is the remainder of $\left|g_{n m l}(\mathbf{k}, \mathbf{q})\right|^{2}$ after removing the long-range contribution $\left|g_{n m /}^{L}(\mathbf{k}, \mathbf{q})\right|^{2} 47$. By setting $E$ at Fermi energy $E_{F}$ and considering only small $\omega$, it reduces to the conventional isotropic Eliashberg function for metals. Since $\left|g_{n m l}(\mathbf{k}, \mathbf{q})\right|^{2} / \omega_{\text {/q }}$ varies slowly as discussed above, this expression converges fast with increasing Brillouin zone sample density. In the actual first-principles calculations, the delta functions are approximated as Gaussian with broadening of $0.2 \mathrm{eV}$ for electron energy and $0.005 \mathrm{eV}$ for phonon energy, according to our convergence test as given in Supplementary Fig. 1 where converged results were found to be insensitive to smearing parameters. We have also considered the smearing-free tetrahedron method for the integration, however we found that when band edge is not directly sampled by the $\mathbf{k}$-mesh, $a_{1}^{2} F(E, \omega)$ near band edge is significantly underestimated using tetrahedron integration, as shown in Supplementary Fig. 2. So, it is not well-suited for the target of this study and we use the smearing-based method.

The evaluation of $a_{1}^{2} F(E, \omega)$ is very fast after obtaining electron-phonon matrix elements using density functional perturbation theory. The computational cost roughly scales as $N_{E P I C} \sim 3 N_{a t} N_{q} N_{k} N_{b n d}^{2} N_{E} N_{\omega}$, where $N_{a t}, N_{q}, N_{k}, N_{b n d}, N_{E}$, and $N_{\omega}$ are the numbers of atoms, irreducible q points, irreducible $\mathbf{k}$ points, relevant bands, energy sample points, and frequency sample points. $N_{E} N_{\omega}$ is typically in the order of $10^{5}$. For comparison, EPW interpolation roughly scales as $3 N_{a t} N_{q}\left(N_{W F} N_{k}\right)^{2} N^{\text {fine }} N_{k}^{\text {fine }} N_{\text {bnd }}$ where $N_{W F}$ is the number of Wannier functions and $N_{q}^{\text {fihe }} N_{k}^{\text {fine }}$ is the total number of $(\mathbf{k}, \mathbf{q})$ pairs to be interpolated. As shown in previous works, $N_{q}^{\text {fine }} N_{k}^{\text {fine }}$ can be as large as over $10^{10}$ for silicon ${ }^{9}$ and even higher for $\mathrm{GaAs}^{14-16}$. Comparison of computational costs by EPIC STAR and EPW are given in Supplementary Information.

The energy-dependent scattering rate from phonon branch I simply becomes

$$
\begin{aligned}
\tau_{l}^{-1}(E, \mu, T)= & 2 \pi\left\{\int_{0}^{+\infty} a_{l}^{2} F(E, \omega)[f(E+\hbar \omega-\mu, T)+n(\hbar \omega, T)] d \omega\right. \\
& \left.+\int_{-\infty}^{0} a_{l}^{2} F(E, \omega)[1-f(E+\hbar \omega-\mu, T)+n(\hbar \omega, T)] d \omega\right\}
\end{aligned}
$$

For polar semiconductors like III-V compounds, the electron-POP coupling matrix diverges around $\Gamma$ point, as described by Fröhlich model ${ }^{40,57}$. The analytical ab initio description based on Born effective charge, dielectric tensor, and phonon dispersion from DFPT calculations has been proposed recently ${ }^{47,48}$. Such strong electron-POP interaction often dominates the scattering as demonstrated using state-of-the-art electron-phonon coupling method for $\mathrm{GaAs}^{14-16,48}$, anatase $\mathrm{TiO}_{2}{ }^{47}$, two-dimensional transition metal chalcogenides ${ }^{58}$, etc.
The piezoelectric scattering from polar acoustic phonons usually contributes at low temperatures ${ }^{39}$, so we will focus on POP scattering here. Such electron-POP coupling matrix divergence significantly increases the difficulty of scattering rate evaluation as very dense $\mathbf{q}$ mesh needs to be sampled ${ }^{14-16}$. Therefore, we take advantage of the analytical expression of such POP scattering matrix elements to simplify the computation. Here, we take the average electron-POP coupling matrix $\frac{i C_{\mathrm{POP}, l}}{|\mathbf{q}|}$ for each phonon branch I where $C_{P O P, I}^{2}$ is the angular averaged Fröhlich coupling strength given by an integration over a solid angle

$$
C_{\mathrm{POP}, I}^{2}=\lim _{\mathbf{q} \rightarrow 0} \int|\mathbf{q}|^{2}\left|g_{l, \mathbf{q}}^{L}\right|^{2} \frac{d \hat{q}}{4 \pi}
$$

Here $g_{l, \mathbf{q}}^{L}$ is the ab initio long-range contribution to the intraband electron-phonon interaction as defined in ref. ${ }^{47}$ and $\hat{q}$ is unit vector along q. For acoustic phonons, this term vanishes due to translational symmetry. For transverse optical phonons, it also vanishes as polarization is orthogonal to momentum q. This leaves only LO branches with non-zero $C_{\text {POP.I. The POP contribu- }}^{2}$ tion from phonon branch $/$ is then ${ }^{39}$

$$
\begin{aligned}
\tau_{\mathrm{POP}, I}^{-1}(E)= & C_{\mathrm{POP}, I}^{2} \frac{\Omega}{\pi \hbar^{2}} \sqrt{\frac{m_{b}(E)}{2 E}}\left\{\sinh ^{-1} \sqrt{\frac{E}{\hbar \omega_{l}}}\left[n\left(\hbar \omega_{l}, T\right)+f\left(E+\hbar \omega_{l}-\mu, T\right)\right]\right. \\
& \left.+\sinh ^{-1} \sqrt{\frac{E}{\hbar \omega_{l}}-1}\left[n\left(\hbar \omega_{l}, T\right)+1-f\left(E-\hbar \omega_{l}-\mu, T\right)\right]\right\}
\end{aligned}
$$

where $m_{b}(E)$ is the average energy-dependent effective mass from band curvature calculation with the energy $E$ relative to the band edge, $\omega_{l}$ is the average frequency of phonon branch / near $\Gamma$ point, while $\mu$ and $T$ are chemical potential and absolute

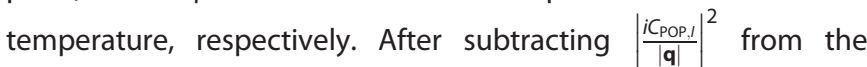
squared electron-phonon coupling matrix $\left|g_{n m l}(\mathbf{k}, \mathbf{q})\right|^{2}$, we use the remainder to evaluate $a_{l}^{2} F(E, \omega)$ and corresponding scattering rate $\tau_{l}^{-1}$. All scattering rate contributions will be added together to calculate the scattering time $\tau(E)$. This information including $C_{\mathrm{POP}, I}$ and $a_{1}^{2} F(E, \omega)$ may also be used to construct a full Boltzmann equation for direct iterative solution ${ }^{9,14}$, but we will focus on scattering time approximation in this work.

In the presence of free carriers, electrostatic screening effect could be important ${ }^{39}$ which reduces the impurity and POP scattering strength. Taking this effect into account, we include it as screening length $L$ which is defined as $^{38,39}$

$$
L^{-2}=\frac{e^{2}}{\epsilon \varepsilon_{0}} \int\left(-\frac{\partial f}{\partial E}\right) \rho(E) d E
$$

The relative dielectric constant $\epsilon$ is obtained from the same DFPT calculation for phonons ${ }^{59}$. It will be used in the dielectric function $\epsilon(\mathbf{q})=1+L^{-2}|\mathbf{q}|^{-2}$. For heavily doped semiconductors, it is also necessary to consider charged impurities induced by doping. Here we consider the simple yet powerful Brooks-Herring ${ }^{39,49,50}$ model of charged impurity scattering with free carrier screening. The corresponding scattering rate is

$\tau_{\mathrm{imp}}^{-1}(E) \approx \frac{n_{\mathrm{imp}} e^{4}}{16 \sqrt{2 m_{b}(E)} \pi \epsilon^{2} \varepsilon_{0}^{2}} \frac{1}{\sqrt{E^{3}}}\left[\ln \left(1+\frac{8 m_{b}(E) E L^{2}}{\hbar^{2}}\right)-\frac{8 m_{b}(E) E L^{2}}{\hbar^{2}+8 m_{b}(E) E L^{2}}\right]$

\section{Validations and discussions}

Silicon is an extensively studied and widely used high mobility semiconductor. Its room temperature electron intrinsic mobility from experimental measurements ranges from about 1400 to $1800 \mathrm{~cm}^{2} \mathrm{~V}^{-1} \mathrm{~s}^{-160-63}$. The phonon-limited electron mobility of silicon has been modeled using BTE with electron-phonon scattering from $\mathrm{DPT}^{30}$, linear interpolation ${ }^{13}$, and Wannier 

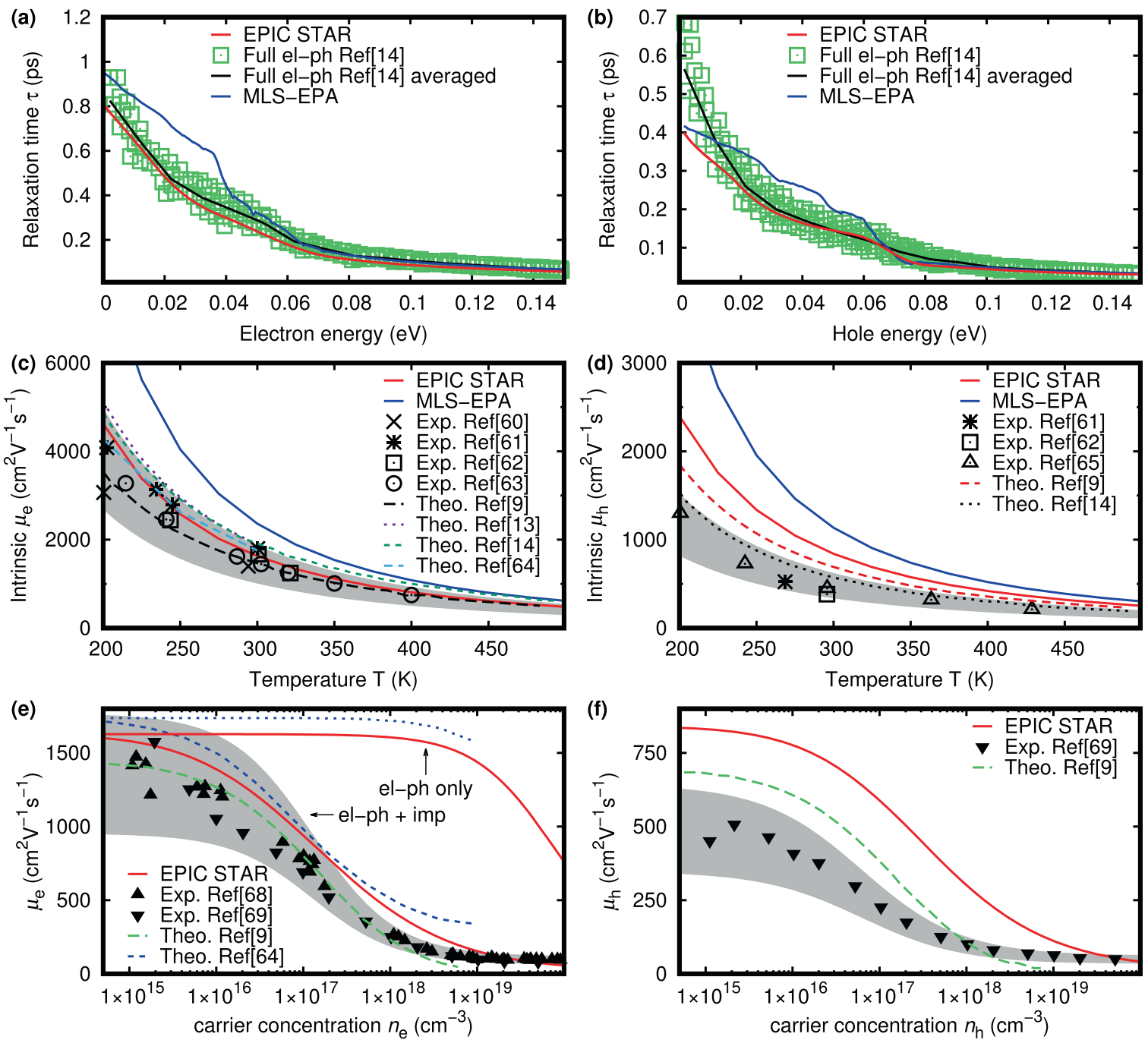

Fig. 1 Electron and hole scattering times and mobilities of Si. Energy dependence of scattering time in silicon for $\mathbf{a}$ electrons and $\mathbf{b}$ holes, calculated using EPIC STAR and MLS-EPA as compared with literature ${ }^{14}$ using full electron-phonon calculations. Temperature-dependent intrinsic mobilities of $\mathbf{c}$ electrons and $\mathbf{d}$ holes, compared with experimental measurements (Exp.) and theoretical predictions (Theo.) in literature. Intrinsic mobilities are calculated with carrier concentration lower than $10^{14} \mathrm{~cm}^{-3}$ to avoid impurity effect. Experimental results are indicated as symbols with crosses ${ }^{60}$, stars $^{61}$, squares ${ }^{62}$, circles $^{63}$, and triangles ${ }^{65}$, Dashed lines are phonon-limited BTE prediction ${ }^{9,13,14,64}$. The region with variation of $\pm 30 \%$ of the power law fitting of temperature dependence with $T^{-2.42}$ and $T^{-2.2}$ for electrons and holes, as defined in ref. ${ }^{67}$ are illustrated in gray. Doping dependences for e n-type electron mobility and $\mathbf{f}$ p-type hole mobility in lightly to heavily doped silicon are compared with experimental measurement and theoretical predictions. Upward-pointing triangles are from ref. ${ }^{68}$ while downwardpointing triangles are from ref. ${ }^{69}$. Dashed lines are first-principles predictions $s^{9,64}$. In heavily doped region, a better agreement is achieved by including ionized impurity scattering (el-ph+imp), which clearly shows the important role of impurities in silicon when doping concentration is increased.

interpolation $^{9,14,64}$ calculations. These full electron-phonon coupling calculations reported intrinsic electron mobility from 1366 to $1915 \mathrm{~cm}^{2} \mathrm{~V}^{-1} \mathrm{~s}^{-1}$, depending on the accuracy of band structure, lattice constant, and Brillouin zone sampling density. The temperature dependence of mobility is also accurately reproduced by phonon-limited Boltzmann transport for a wide range of temperatures, while the effect of doping can also be well described with inclusion of impurity scattering in Brooks-Herring model $^{9}$. The general agreement between experimental measurement and theoretical prediction demonstrates the predictive power of BTE. Therefore, it would be useful to compare the carrier mobility of silicon calculated using EPIC STAR with those in the literature for validation of this fast and simplified method.

First, we calculated the phonon-limited scattering time for silicon using both EPIC STAR and MLS-EPA, and compared them with results from literature as shown in Fig. 1a, b. As expected, EPIC STAR shows good agreement especially for low energy carriers, where optical phonon emission is forbidden by energy conservation and acoustic phonon scattering is important. This accordance is attributed to the explicit $\omega$-dependence of $a_{1}^{2} F(E, \omega)$ which should be more accurate for the rapidly varying acoustic phonon frequency, instead of a single $\omega$ for the whole acoustic phonon branch in MLS-EPA. For carriers with higher energy, both EPIC STAR and MLS-EPA agree well with results from full electron-phonon coupling calculations using EPW and from other published works ${ }^{13,14}$.

Then, we calculated the intrinsic electron (hole) mobility defined as $\boldsymbol{\mu}_{e(h)}=\boldsymbol{\sigma} / e n_{e(h)}$, with $n_{e(h)}$ being $\mathrm{n}$-(p-)type carrier concentration which is lower than $10^{14} \mathrm{~cm}^{-3}$, such that impurity effect is negligible. The calculated intrinsic mobilities are compared with experimental measurement for high purity silicon with temperatures from 200 to $500 \mathrm{~K}^{60-63,65}$, and theoretical predictions using phonon-limited BTE as shown in Fig. 1C, $d^{9,13,14,64}$. The electron mobility calculated using EPIC STAR is in 
excellent agreement with both experimental and theoretical results in literature, while MLS-EPA overestimates the mobility. Phonon-limited BTE prediction in literatures produced electron mobility from 1366 to $1915 \mathrm{~cm}^{2} \mathrm{~V}^{-1} \mathrm{~s}^{-1}$ at $300 \mathrm{~K}$, while EPIC STAR predicts it to be $1637 \mathrm{~cm}^{2} \mathrm{~V}^{-1} \mathrm{~s}^{-1}$. The hole mobility is overestimated in all theoretical predictions as compared with experimental values, which could be ascribed mainly to the significant underestimation of heavy hole effective mass. The heavy hole effective mass along [100] direction extracted from experiments is $0.46 m_{e}$ in ref. ${ }^{66}$ and $0.55 m_{e}$ in ref. ${ }^{65}$ while firstprinciples predictions, including both LDA/GGA and GW calculations ${ }^{9,14}$, are only half of the experimental values. Detailed analyses of the role of density functionals, GW corrections, spin-orbit couplings and electron-phonon renormalization are presented in ref. ${ }^{9}$ where the hole mobility at $300 \mathrm{~K}$ using band structure from first-principles varies from 658 to $820 \mathrm{~cm}^{2} \mathrm{~V}^{-1} \mathrm{~s}^{-1}$. In that work, a better agreement was achieved using experimental effective mass, resulting in hole mobility of $502 \mathrm{~cm}^{2} \mathrm{~V}^{-1} \mathrm{~s}^{-1}$ as compared with experimental value of $450 \mathrm{~cm}^{2} \mathrm{~V}^{-1} \mathrm{~s}^{-167}$. EPIC STAR calculation using LDA without spin-orbit coupling predicts hole mobility of $840 \mathrm{~cm}^{2} \mathrm{~V}^{-1} \mathrm{~s}^{-1}$, achieving agreement with full electron-phonon coupling calculation at a much lower computational cost. And the difference with experimental value mainly comes from hole effective mass and dielectric constant discrepancy between DFT band structure and experimental value ${ }^{9}$. By using experimental hole effective mass, we obtain a hole mobility of $660 \mathrm{~cm}^{2} \mathrm{~V}^{-1} \mathrm{~s}^{-1}$ at $300 \mathrm{~K}$, and after rescaling the electron-phonon coupling using experimental dielectric constant following ref. ${ }^{9}$ it further reduces to $520 \mathrm{~cm}^{2} \mathrm{~V}^{-1} \mathrm{~s}^{-1}$ which is very close to the experimental value. This observation suggests that an accurate DFT band structure is important for reliably predicting the charge transport properties. The carrier mobilities in heavily doped cases are also investigated, as shown in Fig. 1e, f. At high doping level, inclusion of impurity scattering is necessary to reproduce experimental trend ${ }^{68,69}$ where mobility decreases significantly as more dopants are introduced.

The full electron-phonon BTE calculations require very dense sampling in both electron and phonon Brillouin zone. Previous works using linear interpolation ${ }^{13}$ and Wannier interpolation ${ }^{14}$ show that converged results are achieved using uniform $\mathbf{k}$ - and qgrids with $10^{6}$ points in the electron and phonon Brillouin zone. With symmetry considerations and random sampling, a smaller number of inequivalent points, i.e., $8.5 \times 10^{4} \mathbf{k}$-points and $2 \times 10^{5}$ q-points would be sufficient ${ }^{9}$. However, this is still a large number as every ( $\mathbf{k}, \mathbf{q})$ pair needs to be sampled. To estimate the computational cost, we investigated the total number of CPU hours consumed by EPIC STAR and EPW respectively, using the same computer with 2 Intel Xeon E5-2690 v3 CPUs. DFPT calculation using QUANTUM ESPRESSO, which is needed for both EPIC STAR and EPW, took 6 CPU hours with $6^{3}$ q-points. The evaluation of electron-phonon matrix in Wannier basis took 112 CPU hours. And a subsequent Wannier interpolation from $6^{3} \mathbf{q}$ points and $12^{3} \mathbf{k}$-points to $40^{3} \mathbf{q}$-mesh and 3322 irreducible $\mathbf{k}$ points (reduced from $40^{3}$ uniform grid) using $\mathrm{EPW}^{8}$ was performed for speed test. With very small interpolated sampling in Brillouin zone, it still took 322 CPU hours which is much longer than the DFPT calculation itself. With a denser $8.5 \times 10^{4} \mathbf{k}$-points and $2 \times$ $10^{5}$ q-points, the hours could be further increased by orders of magnitude. For comparison, EPIC STAR calculation based on the same DFPT calculation took less than 2 CPU hours to obtain $a_{1}^{2} F$, $\omega_{1}, v_{a \beta}^{2}(E)$, and $\rho(E)$, and the subsequent scattering time and BTE calculations required only a few seconds. So, the total CPU time used for EPW calculation on a small fine grid, which is 440 CPU hours, is much longer than the total time of 8 CPU hours for EPIC STAR calculation. And the difference would be even larger to reach convergence for EPW. In addition, if the Wannierization is not done correctly, interpolation may result in dissimilar band structures especially in cases with band entanglements ${ }^{70,71}$, so it may not be trivial to automate the process for arbitrary materials. EPIC STAR, on the other hand, uses Fourier interpolation as implemented in BoltzTraP which does not require such information, and can be interpolated from a much denser initial k-grid at low cost. In combination with order-of-magnitude lower computational cost, EPIC STAR enables fast and reliable prediction of phonon- and impurity-limited charge transport properties.

Polar optical phonon scattering, which is important in polar materials such as III-V compounds like GaAs ${ }^{14-16,48}$, is also treated in EPIC STAR. Due to the diverging behavior of electron-POP coupling matrix elements, direct Brillouin zone integration methods like EPW requires an even denser q-point sampling for such materials as compared to non-polar materials. A uniform $\mathbf{q}$ grid of $10^{6}-10^{7}$ points is needed for electron scattering rate in GaAs, while extremely fine k-points over $10^{7}$ are needed for determining charge transport properties like carrier mobility ${ }^{14-16}$. First-principles transport properties prediction of polar semiconductors is therefore difficult. Via EPIC STAR, we are able to provide a workaround by calculating the energy-dependent scattering rates using analytical expression. DFPT and EPIC STAR calculation using a coarse q-grid of $6^{3}$ takes $50 \mathrm{CPU}$ hours. Using EPW to interpolate from the same coarse grid to $80^{3}$ q-points and 23,842 irreducible k-points (reduced from an $80^{3}$ uniform mesh), it took 767 CPU hours which is longer by over 1 order of magnitude. Since full EPW calculation for GaAs requires much more $\mathbf{k}$ - and qpoints ${ }^{14}$, the actual difference should be even larger.

As shown in Fig. 2a, while EPIC STAR gives quantitative agreement with full electron-phonon calculations in literature ${ }^{14-}$ 16 , the MLS-EPA results deviate significantly from them. This is a natural consequence of the absence of POP scattering, which dominates the electron scattering in $\mathrm{GaAs}$ and is greater than the scattering from acoustic and non-polar optical phonons by almost an order of magnitude near the band edge. While the experimentally measured electron mobility varies from $0.7 \times 10^{4}$ to $1 \times 10^{4} \mathrm{~cm}^{2} \mathrm{~V}^{-1} \mathrm{~s}^{-1}$ at $300 \mathrm{~K}^{72-76}$, their theoretical results range from $0.7 \times 10^{4}$ to about $1.2 \times 10^{4} \mathrm{~cm}^{2} \mathrm{~V}^{-1} \mathrm{~s}^{-114-16}$, with energy scattering time approximation results being lower than the iterative solution of BTE. Thus, EPIC STAR prediction of $9770 \mathrm{~cm}^{2}$ $\mathrm{V}^{-1} \mathrm{~s}^{-1}$, at a much smaller cost, shows general agreement with both experimental measurements and previous theoretical works which used accurate yet more expensive methods by direct integration of the Brillouin zone.

In addition to carrier mobility, EPIC STAR can also predict other transport coefficients from BTE, including electrical conductivity, Seebeck coefficient, and electronic contribution to thermal conductivity. Here, we use EPIC STAR to study the thermoelectric performance of $\mathrm{Mg}_{2} \mathrm{Si}$. $\mathrm{Mg}_{2} \mathrm{X}(\mathrm{X}=\mathrm{Si}, \mathrm{Ge}, \mathrm{Sn})$ is a family of high performance thermoelectric materials which do not comprise expensive and less abundant elements like $\mathrm{Bi}$ or $\mathrm{Te}$, or toxic elements like $\mathrm{Pb}^{77}$. Simple $\mathrm{Mg}_{2} \mathrm{X}$ such as n-type $\mathrm{Mg}_{2} \mathrm{Si}$ is able to achieve $z T$ of $0.86^{78}$, while higher $z T$ has been realized using solid solutions of different $X$ through band convergence. For example, $z T$ of $\mathrm{Mg}_{2.15} \mathrm{Si}_{0.28} \mathrm{Sn}_{0.71} \mathrm{Sb}_{0.006}$ can reach up to 1.3 at $700 \mathrm{~K}^{22}$, and that of $\mathrm{Mg}_{2.16}\left(\mathrm{Si}_{0.3} \mathrm{Ge}_{0.05} \mathrm{Sn}_{0.65}\right)_{0.98} \mathrm{Sb}_{0.02}$ with 1.45 at $750 \mathrm{~K}^{79}$.

The electron mobilities of doped $\mathrm{Mg}_{2} \mathrm{Si}$ were first studied and compared with those in literature ${ }^{80}$. The calculation was performed with the same doping concentration as the experiments, from which good agreement has been achieved between EPIC STAR and experimental measurement for a wide temperature range as shown in Fig. 3a.

Bi-doped $\mathrm{Mg}_{2} \mathrm{Si}$ with different doping levels have been experimentally studied with Bi: $\mathrm{Mg}_{2} \mathrm{Si}$ ratio from 0.1 to $2 \%{ }^{78}$. The highest $z T$ of 0.86 was realized at $862 \mathrm{~K}$ with $2 \% \mathrm{Bi}$ doping. Here, we performed EPIC STAR calculations for each doping level studied experimentally and compared the resulting electrical conductivity, Seebeck coefficient, and figure of merit $z T$ with the experimental measurements. The carrier concentrations are fixed to the experimental value at $300 \mathrm{~K}$ from Hall measurements ${ }^{78}$. 

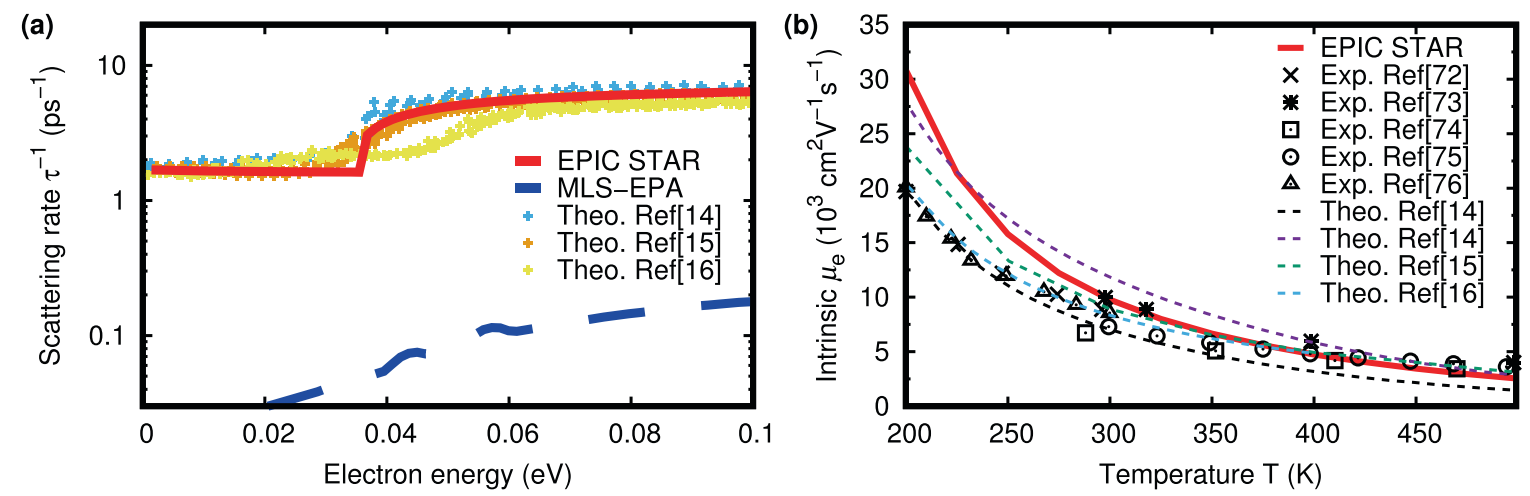

Fig. 2 Electron scattering time and mobility of GaAs. a Energy dependence of scattering time for electrons in GaAs, calculated using EPIC STAR and MLS-EPA, as compared with refs. ${ }^{14-16}$ using full electron-phonon calculation which is much more computationally expensive. EPIC STAR achieves comparable results for both scattering rate and intrinsic mobility compared to full electron-phonon calculation, with at least 1 order of magnitude faster computation speed. Due to the absence of POP scattering, the MLS-EPA results (blue dashed line) deviate substantially from the full electron-phonon calculations. Solid red line is purely first-principles EPIC STAR results. The sharp increase in scattering rate near $0.04 \mathrm{eV}$ marks the onset of POP emission process. b Temperature-dependent intrinsic mobility of electrons in GaAs, compared with experimental measurements (Exp.) and full first-principles theoretical predictions (Theo.) in literature. The experimental results are indicated as crosses $^{72}$, stars ${ }^{73}$, squares ${ }^{74}$, circles $^{75}$, and triangles ${ }^{76}$. Thin dashed lines are phonon-limited BTE prediction with energy scattering time approximation (STA) and with iterative solution of BTE ${ }^{\text {i4-16. }}$
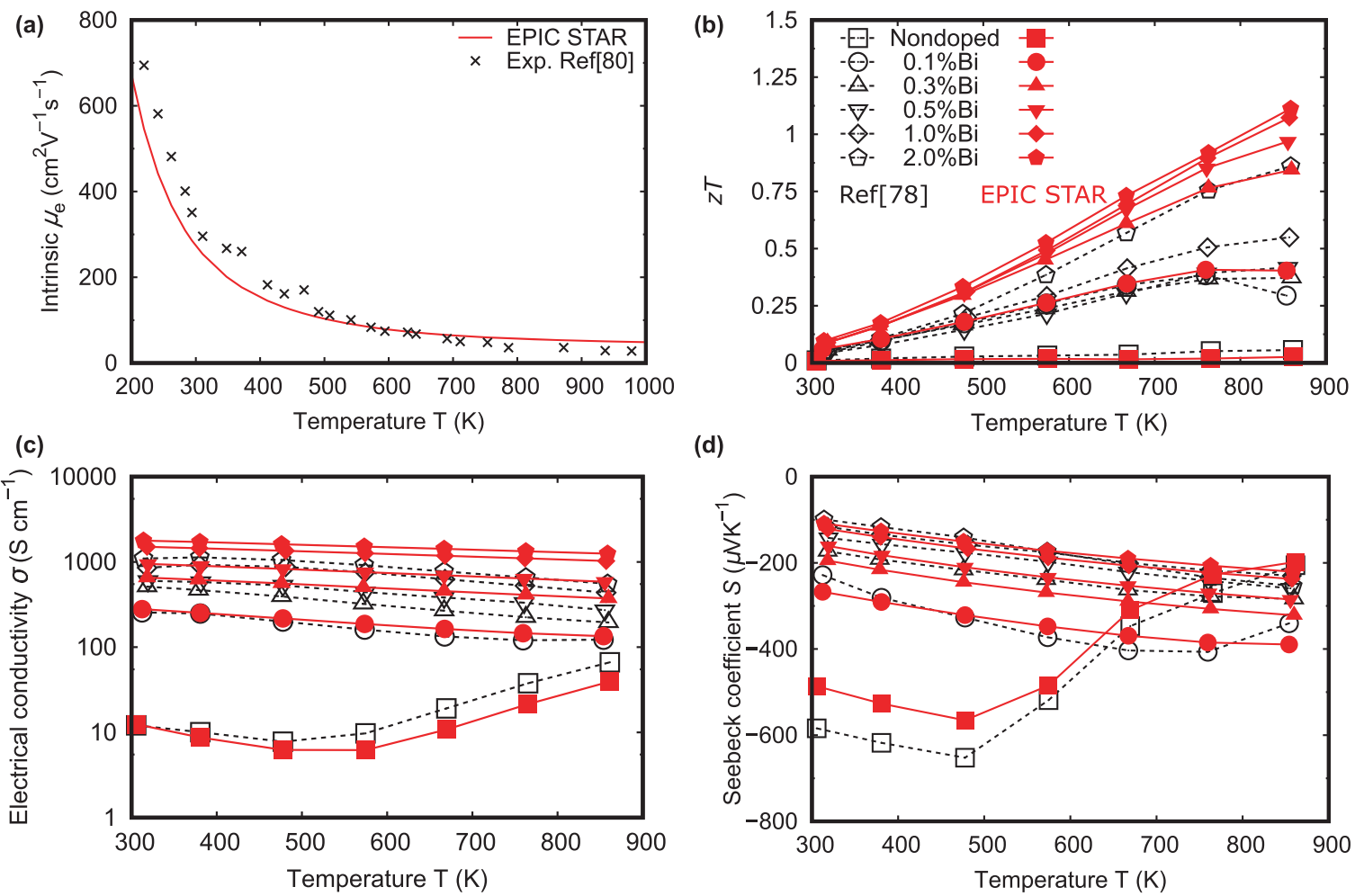

(d)

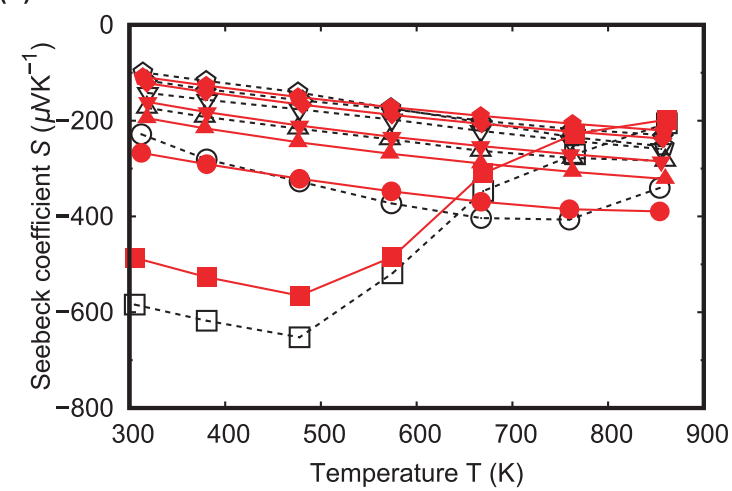

Fig. 3 Transport properties of $\mathbf{M g}_{2} \mathbf{S i}$. a Electron mobility calculated at different temperature, as compared with experimental measurement by Morris et al. ${ }^{80}$. Predicted values for $\mathbf{b}$ figure of merit $z T$, c electrical conductivity, and $\mathbf{d}$ Seebeck coefficients of Bi-doped Mg ${ }_{2} \mathrm{Si}$ are compared with previous experimental measurement ${ }^{78}$.

Since the PBE GGA band gap of $0.22 \mathrm{eV}$ is an underestimation which will affect the predicted value of Seebeck coefficient for low doping levels, we instead used the TB-mBJ meta-GGA ${ }^{81}$ band gap of $0.6 \mathrm{eV}$ from ref. ${ }^{82}$ by performing a rigid shift of conduction band. The lattice contribution to thermal conductivity $K_{L}$, which is beyond the scope of this study, is taken from experimental data which was estimated based on Wiedemann-Franz law where Lorenz number was assumed to be $2.45 \times 10^{-8} \mathrm{~V}^{2} \mathrm{~K}^{-2} 78$. However, the actual Lorenz number may differ from this value and depends on both doping and temperature ${ }^{37}$. Therefore, the figure of merit $z T$ here is just an estimation. The calculated $z T$, electrical conductivity, and Seebeck coefficient are compared with experimental measurements in ref. ${ }^{83}$ as shown in Fig. 3b-d. The predicted intrinsic mobility and thermoelectric performance agrees with experimental measurements along a wide temperature range. This demonstrates the predictive power of our approach for both intrinsic and doped semiconductors.

Half-Heusler $(\mathrm{HH})$ alloys constitute an important class of high power factor TE materials ${ }^{84,85}$. Among many $\mathrm{HH}$ compounds, $\mathrm{NbFeSb}$ has been proposed as one of the promising candidates in 
(a)

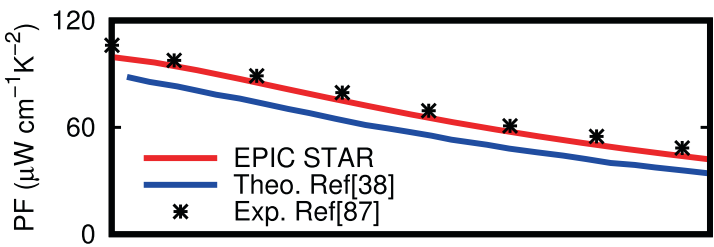

(b)

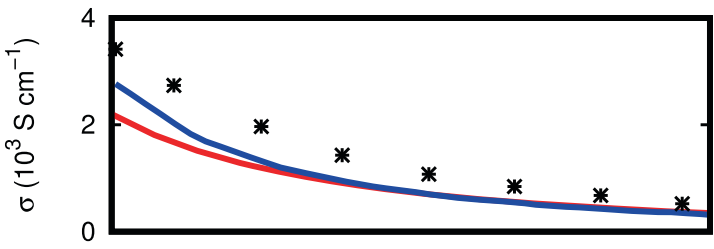

(c)

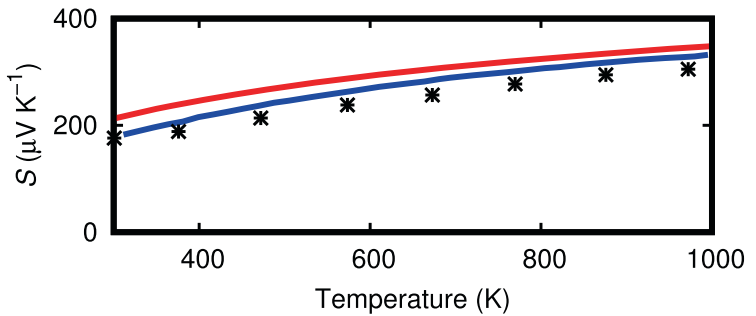

Fig. 4 p-type NbFeSb thermoelectric performance. Thermoelectric performance of p-type NbFeSb, including a power factor, $\mathbf{b}$ electrical conductivity, and c Seebeck coefficient from EPIC STAR prediction from 300 to $1000 \mathrm{~K}$, compared with experimental measurement of Ti-doped $\mathrm{NbFeSb}^{87}$ and EPW prediction in literature ${ }^{38}$. Calculations were performed at a fixed hole-doping concentration of $2.0 \times 10^{20}$ $\mathrm{cm}^{-3}$ for all temperatures.

a theoretical study in $2008^{86}$ using BTE in CSTA. Later in 2014 it has been confirmed as a good TE material with $z T \sim 1$ at $700^{\circ} \mathrm{C}$ in a combined experimental and theoretical study using $\mathrm{EPA}^{2}$. A record-high room temperature power factor of $106 \mu \mathrm{Wcm}{ }^{-1} \mathrm{~K}^{-2}$ was achieved with Ti-doped NbFeSb, and it has been suggested that electron-phonon interaction is the dominant charge carrier scattering mechanism ${ }^{87}$. A very recent theoretical study using accurate EPW method successfully reproduced NbFeSb TE performance for a wide range of temperatures, and indicates that the high power factor of $\mathrm{HH}$ alloys mainly arises from weak electron-acoustic-phonon scattering which comes from the nonbonding feature of electron orbitals ${ }^{38}$. The extensive experimental and theoretical study of NbFeSb provides another good candidate for EPIC STAR validation, and our approach indeed achieves excellent agreement with both experimental measurement and EPW prediction from 300 to $1000 \mathrm{~K}$. As shown in Fig. 4, EPIC STAR predicts a power factor of $99 \mu \mathrm{Wcm}^{-1} \mathrm{~K}^{-2}$, comparable to experimentally measured $106 \mu \mathrm{Wcm}^{-1} \mathrm{~K}^{-2}$ and EPW prediction of around $90 \mu \mathrm{Wcm}^{-1} \mathrm{~K}^{-2} 38$. The Seebeck coefficient is slightly higher than literature while conductivity is lower. This EPIC STAR calculation used a coarse $6^{3} \mathbf{q}$-mesh, which is the same as that in EPW before Wannier interpolation. With EPW, interpolation to $40^{3}$ k- and q-points needs $497 \mathrm{CPU}$ hours, while $80^{3}$ is needed to reach converged results ${ }^{38}$, which requires at least thousands of CPU hours based on previous estimation. However, DFPT calculation took $145 \mathrm{CPU}$ hours and the subsequent EPIC-STAR calculation only took less than 5 CPU hours to obtain comparable results, reducing time by at least 1 order of magnitude. And the intrinsic hole mobility from EPIC STAR calculation, which is $64 \mathrm{~cm}^{2}$ $\mathrm{V}^{-1} \mathrm{~s}^{-1}$, is comparable to $77 \mathrm{~cm}^{2} \mathrm{~V}^{-1} \mathrm{~s}^{-1}$ from EPW calculation ${ }^{38}$ while EPA gives a significant overestimation of $149 \mathrm{~cm}^{2} \mathrm{~V}^{-1} \mathrm{~s}^{-1}$ which comes from the absence of POP scattering. The results from EPA on p-type NbFeSb is given in Supplementary Information. This again emphasizes the efficiency and reliability of EPIC STAR which is expected to be generally applicable for a broader range of materials.
In addition to the validation of the above theoretical and experimental results in the literature, we also apply this method to predict the thermoelectric performance of $\mathrm{NaInSe}_{2}$ which has not been reported yet. It has been synthesized experimentally whereby a layered anisotropic crystal structure was found, as shown in Supplementary Fig. $2^{88}$. It possesses a similar crystal structure to $\mathrm{NaCoO}_{2}$, of which its thermal conductivity is greatly suppressed due to rattling modes in the presence of Na vacancy ${ }^{89}$. Although $\mathrm{Na}$ ions can be rattling in the presence of vacancy, its ionic contribution to charge transport is very small in similar structures $^{90}$, so electronic transport is expected to dominate the electrical properties. Reduced thermal conductivity is desirable for thermoelectric applications, and therefore, its electronic part of thermoelectric performance is explored in this work.

First, we performed EPW calculation to validate our calculation of scattering times. The case for $\mathrm{NaInSe}_{2}$ is complicated because of its complex electronic band structure and phonon dispersion as shown in Fig. $5 \mathrm{a}, \mathrm{b}$. The lowest conduction valley at $\Gamma$ point is energetically very close to the second lowest triply degenerate $F$ valley which is only slightly more positive by $0.1 \mathrm{eV}$, and they possess very different effective masses. The valence band has a strong effective mass anisotropy which is ten times heavier along the $z$ direction than the in-plane directions (see Supplementary Fig. 2 for the definitions). The phonon dispersion is also different along in-plane and out-of-plane directions as shown in Fig. 5b. The electron-phonon scattering as illustrated in Fig. $5 \mathrm{c}$ is dominated by polar longitudinal optical phonons even for high energy electrons at $0.5 \mathrm{eV}$ above conduction band edge. This again emphasizes the importance of correct treatment for POP scattering in electron-phonon scattering time prediction. By comparing EPIC STAR scattering time with averaged EPW scattering time for carriers with the same energy, we found good agreement even for such a complicated situation, as shown in Fig. $5 d$.

With such a complex electronic band structure, the Fermi Surface Complexity Factor $N_{v}^{*} K^{*}=\left(\frac{m_{s}^{*}}{m_{c}^{*}}\right)^{\frac{3}{2}}$ proposed by Gibbs et al. $^{24}$ should be large and be correlated with good thermoelectric performance. Here, we use a slightly modified definition for anisotropic material by distinguishing the in-plane $\left(m_{\|}^{*}\right)$ and out-of-plane transport effective mass $\left(m_{\perp}^{*}\right)$. The $N_{v}^{*} K^{*}$ value for $n$ type $\mathrm{NalnSe}_{2}$ is found to be 2.8 for in-plane and 1.8 for out-ofplane transport, while for p-type it is 7.6 for in-plane and 0.7 for out-of-plane. The peak n-type power factor predicted using EPIC STAR at $300 \mathrm{~K}$ is $\mathrm{PF}_{\|}=13.4 \mu \mathrm{Wcm}^{-1} \mathrm{~K}^{-2}$ and $\mathrm{PF}_{\perp}=8.5 \mu \mathrm{Wcm}^{-1} \mathrm{~K}^{-2}$, while for $\mathrm{p}$-type $\mathrm{PF}_{\|}=28.4 \mu \mathrm{Wcm}^{-1} \mathrm{~K}^{-2}$ and $\mathrm{PF}_{\perp}=2.4 \mu \mathrm{Wcm}{ }^{-1} \mathrm{~K}^{-2}$, respectively. At low temperature, $\mathrm{p}$ type $\mathrm{NalnSe}_{2}$ achieves higher PF due to large power factor from complex band structure. However, as temperature increases, ntype performance increases rapidly and outperforms p-type after $600 \mathrm{~K}$. This is because of increased effective valley degeneracy due to electrons in F-valleys which have a 3-fold degeneracy. F-valleys are $0.1 \mathrm{eV}$ higher than the single $\Gamma$-valley so they are important only when temperature is high enough. The relative trend of PF is in line with $N_{v}^{*} K^{*}$ which is expected as the high anisotropy and valley degeneracy lead to enhanced Seebeck coefficients. However, the CSTA result using a single scattering time $\tau_{\text {CSTA }}=4.5 \mathrm{fs}$, which albeit fits the peak p-type $\mathrm{PF}_{\|}$, results in a serious underestimation of a factor of 3 in n-type power factors. Moreover, the optimal p-doping level from CSTA prediction is also different from EPIC STAR result. It is also observed that CSTA PF increases monotonically as temperature rises, as shown in Supplementary Fig. 3, which differs significantly from EPIC STAR calculation where PF decreases at high temperature due to stronger electron-phonon scattering (Fig. 5f). This again emphasizes the importance of reliable calculation of scattering times.

In conclusion, we proposed a swift and automation-friendly approach for intrinsic and impurity-limited charge transport 

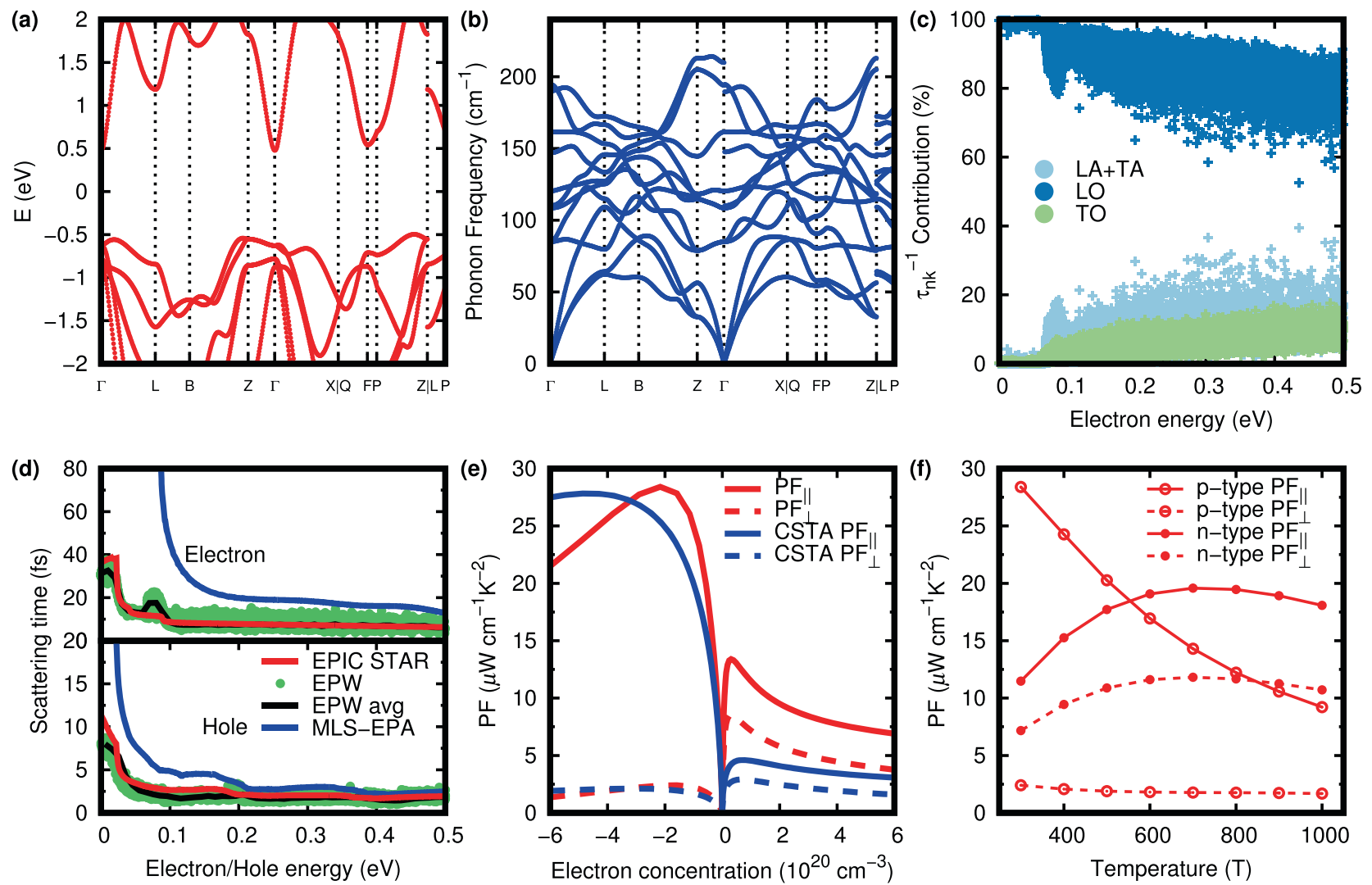

Fig. 5 Band structures and properties of $\mathrm{NaInSe}_{2}$. a Electronic band structure of $\mathrm{NalnSe}_{2}$. b Phonon dispersion of NalnSe $\mathrm{P}_{2}$. The observed longitudinal and transverse optical phonon (LO-TO) splitting and non-analytical behavior demonstrates the polar semiconductor nature of $\mathrm{NalnSe}_{2}$. c Mode-resolved electron-phonon scattering rate contribution from longitudinal and transverse acoustic (LA + TA), longitudinal optical (LO), and transverse optical (TO) phonons. The polar LO branches contribute more than $80 \%$ of the scattering rate in a wide energy range. $\mathbf{d}$ Electron and hole scattering time calculated using EPW (symbols) and their average (black lines), MLS-EPA (blue lines), and EPIC STAR (red lines). The EPIC STAR is in agreement with averaged EPW results from the band edge to the high energy region while the EPA results show larger discrepancy mainly due to the absence of POP scattering. e The predicted doping-dependent power factors at $300 \mathrm{~K}$ compared with CSTA results with $\tau_{\mathrm{CSTA}}=4.5 \mathrm{fs}$. A single scattering time (in the case for CSTA) is clearly not enough for the prediction of both electron and hole, and the peak power factor position is also different from phonon-limited study. $f$ The predicted temperature-dependent power factor at fixed hole $\left(2 \times 10^{20} \mathrm{~cm}^{-3}\right)$ and electron $\left(1 \times 10^{20} \mathrm{~cm}^{-3}\right)$ concentration. $\mathrm{p}$-Type power factor is higher at relatively low temperatures, while $\mathrm{n}$ type power factor peaks around $700 \mathrm{~K}$.

property prediction from first-principles. By introducing generalized Eliashberg function and adding polar optical phonon contribution, impurity scattering, and free carrier screening, the new approach achieves high fidelity especially for both non-polar and polar semiconductors with very small computational cost after DFPT calculations. We demonstrated the importance of polar optical phonon scattering, which suggests that care should be taken when the electronic properties of polar semiconductors are studied without polar optical phonon scattering. We verified our approach by comparing with previous experimental and theoretical results for $\mathrm{Si}, \mathrm{GaAs}, \mathrm{Mg}_{2} \mathrm{Si}$, and $\mathrm{NbFeSb}$, and also revealed $\mathrm{NalnSe}_{2}$ as a potential new TE material. As high-throughput DFPT computations have been demonstrated recently ${ }^{91,92}$, our methodology can be widely applied for reliable high-throughput screening of high mobility semiconductors and highperformance thermoelectric and photovoltaic materials.

\section{METHODS}

Transport coefficients from Boltzmann transport equation Semi-classical Boltzmann transport equation (BTE) could be approximately solved in scattering time approximation, leading to the electronic transport integral tensor of order $p$ as ${ }^{12}$

$$
\left[\mathbf{K}^{p}\right]_{a \beta}=\int_{-\infty}^{+\infty}\left[\sum_{n} \int_{B Z} v_{n \mathbf{k}}^{a} v_{n \mathbf{k}}^{\beta} \tau_{n \mathbf{k}} \delta\left(E-\varepsilon_{n \mathbf{k}}\right) \frac{d \mathbf{k}}{\Omega_{B Z}}\right](E-\mu)^{p}\left[-\frac{\partial f^{0}(E, \mu, T)}{\partial E}\right] d E
$$

Here, for each state $n \mathbf{k}, v_{n \mathbf{k}}^{a}$ is the $a$-direction component of group velocity, $\tau_{n \mathbf{k}}$ is the scattering time, $\varepsilon_{n \mathbf{k}}$ is the energy, and $f^{0}(E, \mu, T)$ is the Fermi-Dirac distribution with given chemical potential $\mu$ and temperature $T$. Spin degeneracy is included in the band index $n . \Omega_{\mathrm{BZ}}=\frac{(2 \pi)^{3}}{\Omega}$ is the Brillouin zone volume where $\Omega$ is the unit cell volume.

The electrical conductivity tensor $\boldsymbol{\sigma}$, Seebeck coefficient $\boldsymbol{S}$, and electronic contribution to thermal conductivity $\boldsymbol{K}_{e}$ can be evaluated using $\mathbf{K}^{p}$ of different orders through ${ }^{12,93}$

$\boldsymbol{\sigma}=\frac{1}{\Omega} e^{2} \mathbf{K}^{0}$

$\mathbf{S}=\frac{1}{-e T}\left[\mathbf{K}^{0}\right]^{-1} \mathbf{K}^{1}$

$\boldsymbol{K}_{e}=\frac{1}{T \Omega}\left[\mathbf{K}^{2}-\mathbf{K}^{1}\left[\mathbf{K}^{0}\right]^{-1} \mathbf{K}^{1}\right]$

Here, $e$ is the elementary charge such that electron charge is $-e$. The carrier mobility can be defined simply as $\boldsymbol{\mu}_{e(h)}=\boldsymbol{\sigma} / e_{e(h)}$ with $n_{e(h)}$ being electron (hole) concentration. The only system-dependent ingredient in this formulation is the so-called transport distribution function ${ }^{93}$ which 
projects the complex electronic state $n \mathbf{k}$-dependence into simple energy E-dependent formula

$$
[\boldsymbol{\Sigma}(E)]_{\alpha \beta}=\sum_{n} \int_{B Z} v_{n \mathbf{k}}^{a} v_{n \mathbf{k}}^{\beta} \tau_{n \mathbf{k}} \delta\left(E-\varepsilon_{n \mathbf{k}}\right) \frac{d \mathbf{k}}{\Omega_{B Z}}
$$

\section{DFT calculations}

All first-principles calculations were performed using QUANTUM ESPRESSO ${ }^{51,52}$ including structure optimization, ground state calculation, and band structure calculations using density functional theory. Phonon and electron-phonon coupling matrix were obtained from DFPT $^{59,94}$ calculation implemented in QUANTUM ESPRESSO. Non-selfconsistent-field band structure calculations were performed on a uniform $48^{3} \mathbf{k}$-mesh and Fourier interpolation into a much denser $240^{3}$ mesh was performed using BoltzTraP in order to obtain the density of states, squared velocity tensor, and energy-dependent effective mass. Local density approximation (LDA ${ }^{95}$ was used for $\mathrm{Si}$ and GaAs for comparison with previous theoretical literature, while generalizedgradient approximation (GGA) with exchange-correlation function given by Perdew, Burke, and Ernzerhof $(\mathrm{PBE})^{96}$ was used for $\mathrm{Mg}_{2} \mathrm{Si}$, $\mathrm{NbFeSb}$, and $\mathrm{NaInSe}_{2}$. Norm-conserving pseudopotentials ${ }^{97,98}$ were used to replace core electrons. Although corrections such as spin-orbit coupling and Hubbard $U$ were not considered in this work, they can be added for all calculations having been implemented and tested for band structure and DFPT calculations in QUANTUM ESPRESSO ${ }^{52}$.

\section{DATA AVAILABILITY}

The authors declare that the data supporting the findings of this study are available within the paper and its Supplementary Information file. Raw data generated by QE are available from the corresponding author G.W. upon request.

\section{CODE AVAILABILITY}

The algorithms presented in this paper have been fully implemented in a FORTRAN package, but the current version is only available from the corresponding author G.W. upon request. After further debugging, the stable version will be released online.

Received: 20 December 2019; Accepted: 20 March 2020; Published online: 07 May 2020

\section{REFERENCES}

1. $\mathrm{Zhu}, \mathrm{H}$. et al. Computational and experimental investigation of $\mathrm{TmAgTe}_{2}$ and $\mathrm{XYZ}_{2}$ compounds, a new group of thermoelectric materials identified by firstprinciples high-throughput screening. J. Mater. Chem. C 3, 10554-10565 (2015).

2. Joshi, G. et al. NbFeSb-based p-type half-Heuslers for power generation applications. Energy Environ. Sci. 7, 4070-4076 (2014).

3. He, R. et al. Enhanced thermoelectric properties of $n$-type NbCoSn half-Heusler by improving phase purity. APL Mater. 4, 104804 (2016).

4. Xi, L. et al. Discovery of high-performance thermoelectric chalcogenides through reliable high-throughput material screening. J. Am. Chem. Soc. 140, 10785-10793 (2018).

5. Brunin, G., Ricci, F., Ha, V. A., Rignanese, G. M. \& Hautier, G. Transparent conducting materials discovery using high-throughput computing. npj Comput. Mater. 5, 1-13 (2019).

6. Bhatia, A. et al. High-mobility bismuth-based transparent p-type oxide from highthroughput material screening. Chem. Mater. 28, 30-34 (2016).

7. Yan, F. et al. Design and discovery of a novel half-Heusler transparent hole conductor made of all-metallic heavy elements. Nat. Commun. 6, 1-8 (2015).

8. Poncé, S., Margine, E. R., Verdi, C. \& Giustino, F. EPW: Electron-phonon coupling, transport and superconducting properties using maximally localized Wannier functions. Comput. Phys. Commun. 209, 116-133 (2016).

9. Poncé, S., Margine, E. R. \& Giustino, F. Towards predictive many-body calculations of phonon-limited carrier mobilities in semiconductors. Phys. Rev. B 97, 121201 (2018).

10. Li, W., Carrete, J., Katcho, N. A. \& Mingo, N. ShengBTE: a solver of the Boltzmann transport equation for phonons. Comput. Phys. Commun. 185 1747-1758 (2014).
11. Carrete, J. et al. almaBTE: a solver of the space-time dependent Boltzmann transport equation for phonons in structured materials. Comput. Phys. Commun. 220, 351-362 (2017).

12. Ziman, J. M. Electrons and Phonons: The Theory of Transport Phenomena in Solids (Oxford University Press, Oxford, 2001).

13. Li, W. Electrical transport limited by electron-phonon coupling from Boltzmann transport equation: an ab initio study of $\mathrm{Si}, \mathrm{Al}$, and $\mathrm{MoS}_{2}$. Phys. Rev. B 92, 075405 (2015).

14. Ma, J., Nissimagoudar, A. S. \& Li, W. First-principles study of electron and hole mobilities of Si and GaAs. Phys. Rev. B 97, 045201 (2018).

15. Zhou, J.-J. \& Bernardi, M. Ab initio electron mobility and polar phonon scattering in GaAs. Phys. Rev. B 94, 201201 (2016).

16. Liu, T.-H., Zhou, J., Liao, B., Singh, D. J. \& Chen, G. First-principles mode-by-mode analysis for electron-phonon scattering channels and mean free path spectra in GaAs. Phys. Rev. B 95, 075206 (2017).

17. Giustino, F., Cohen, M. L. \& Louie, S. G. Electron-phonon interaction using Wannier functions. Phys. Rev. B 76, 165108 (2007).

18. Madsen, G. K. H. \& Singh, D. J. BoltzTraP. A code for calculating band-structure dependent quantities. Comput. Phys. Commun. 175, 67-71 (2006).

19. Madsen, G. K. H., Carrete, J. \& Verstraete, M. J. BoltzTraP2, a program for interpolating band structures and calculating semi-classical transport coefficients. Comput. Phys. Commun. 231, 140-145 (2018).

20. Pizzi, G., Volja, D., Kozinsky, B., Fornari, M. \& Marzari, N. BoltzWann: a code for the evaluation of thermoelectric and electronic transport properties with a maximally-localized Wannier functions basis. Comput. Phys. Commun. 185, 422-429 (2014).

21. Kohn, W. \& Sham, L. J. Self-consistent equations including exchange and correlation effects. Phys. Rev. 140, A1133-A1138 (1965).

22. Liu, W. et al. Convergence of conduction bands as a means of enhancing thermoelectric performance of $\mathrm{n}$-type $\mathrm{Mg}_{2} \mathrm{Si}_{1-x}$ solid solutions. Phys. Rev. Lett. 108 166601 (2012).

23. Pei, Y., Wang, H. \& Snyder, G. J. Band engineering of thermoelectric materials. Adv. Mater. 24, 6125-6135 (2012).

24. Gibbs, Z. M. et al. Effective mass and Fermi surface complexity factor from $a b$ initio band structure calculations. npj Comput. Mater. 3, 8 (2017).

25. Xing, G. et al. Electronic fitness function for screening semiconductors as thermoelectric materials. Phys. Rev. Mater. 1, 065405 (2017).

26. Heremans, J. P., Thrush, C. M. \& Morelli, D. T. Thermopower enhancement in lead telluride nanostructures. Phys. Rev. B 70, 115334 (2004).

27. Wang, S. et al. On intensifying carrier impurity scattering to enhance thermoelectric performance in $\mathrm{Cr}$-doped $\mathrm{Ce}_{\mathrm{y}} \mathrm{Co}_{4} \mathrm{Sb}_{12}$. Adv. Funct. Mater. 25, 6660-6670 (2015).

28. Kang, S. D. \& Snyder, G. J. Charge-transport model for conducting polymers. Nat. Mater. 16, 252-257 (2017).

29. He, M. et al. Thermopower enhancement in conducting polymer nanocomposites via carrier energy scattering at the organic-inorganic semiconductor interface. Energy Environ. Sci. 5, 8351-8358 (2012).

30. Bardeen, J. \& Shockley, W. Deformation potentials and mobilities in non-polar crystals. Phys. Rev. 80, 72-80 (1950).

31. Chen, J., Wang, D. \& Shuai, Z. First-principles predictions of thermoelectric figure of merit for organic materials: deformation potential approximation. J. Chem. Theory Comput. 8, 3338-3347 (2012).

32. Lü, T. Y., Feng, H., Yang, S. W. \& Zheng, J. C. Electronic structures, mechanical properties and carrier mobilities of $\pi$-conjugated $X(X=N i, P d, P t)$ bis (dithiolene) nanosheets: theoretical predictions. Comput. Mater. Sci. 126 170-175 (2017).

33. Shi, W. et al. Poly(nickel-ethylenetetrathiolate) and its analogs: theoretical prediction of high-performance doping-free thermoelectric polymers. J. Am. Chem. Soc. 140, 13200-13204 (2018).

34. Yong, $X$. et al. Tuning the thermoelectric performance of $\pi-d$ conjugated nickel coordination polymers through metal-ligand frontier molecular orbital alignment. J. Mater. Chem. A 6, 19757-19766 (2018).

35. Shi, W. et al. Orbital-engineering-based screening of $\pi$-conjugated $d^{8}$ transitionmetal coordination polymers for high-performance n-type thermoelectric applications. ACS Appl. Mater. Interfaces 10, 35306-35315 (2018).

36. Wang, D., Shi, W., Chen, J., Xi, J. \& Shuai, Z. Modeling thermoelectric transport in organic materials. Phys. Chem. Chem. Phys. 14, 16505 (2012).

37. Samsonidze, G. \& Kozinsky, B. Accelerated screening of thermoelectric materials by first-principles computations of electron-phonon scattering. Adv. Energy Mater. 8, 1800246 (2018).

38. Zhou, J. et al. Large thermoelectric power factor from crystal symmetry-protected non-bonding orbital in half-Heuslers. Nat. Commun. 9, 1721 (2018).

39. Lundstrom, M. Fundamentals of Carrier Transport (Cambridge University Press, Cambridge, 2000). 
40. Giustino, F. Electron-phonon interactions from first principles. Rev. Mod. Phys. 89, 015003 (2017).

41. McMillan, W. L. Transition temperature of strong-coupled superconductors. Phys. Rev. 167, 331-344 (1968).

42. Allen, P. B. \& Dynes, R. C. Transition temperature of strong-coupled superconductors reanalyzed. Phys. Rev. B 12, 905-922 (1975).

43. Grimvall, G. The Electron-Phonon Interaction in Metals (North-Holland Publishing Company, Amsterdam, 1981).

44. Wierzbowska, M., de Gironcoli, S. \& Giannozzi, P. Origins of low- and highpressure discontinuities of $\mathrm{T}_{\mathrm{c}}$ in niobium. Preprint at https://arxiv.org/abs/condmat/0504077 (2005)

45. Koretsune, T. \& Arita, R. Efficient method to calculate the electron-phonon coupling constant and superconducting transition temperature. Comput. Phys. Commun. 220, 239-242 (2017).

46. Giustino, F., Cohen, M. L. \& Louie, S. G. GW method with the self-consistent Sternheimer equation. Phys. Rev. B 81, 115105 (2010).

47. Verdi, C. \& Giustino, F. Fröhlich electron-phonon vertex from first principles. Phys. Rev. Lett. 115, 176401 (2015).

48. Sjakste, J., Vast, N., Calandra, M. \& Mauri, F. Wannier interpolation of the electron-phonon matrix elements in polar semiconductors: polar-optical coupling in GaAs. Phys. Rev. B 92, 054307 (2015).

49. Brooks, H. Theory of the electrical properties of germanium and silicon. Adv. Electron. Electron Phys. 32, 85-182 (1955).

50. Herring, C. Transport properties of a many-valley semiconductor. Bell Syst. Tech. J. 34, 237-290 (1955).

51. Giannozzi, P. et al. QUANTUM ESPRESSO: a modular and open-source software project for quantum simulations of materials. J. Phys.: Condens. Matter 21, 395502 (2009).

52. Giannozzi, P. et al. Advanced capabilities for materials modelling with Quantum ESPRESSO. J. Phys.: Condens. Matter 29, 465901 (2017).

53. Yuan, J. et al. One-dimensional thermoelectrics induced by Rashba spin-orbit coupling in two-dimensional BiSb monolayer. Nano Energy 52, 163-170 (2018).

54. Deng, T. et al. 2D single-layer $\pi$-conjugated nickel bis(dithiolene) complex: a good-electron-poor-phonon thermoelectric material. Adv. Electron. Mater. 5, 1800892 (2019).

55. Shi, W. et al. Unprecedented enhancement of thermoelectric power factor induced by pressure in small-molecule organic semiconductors. Adv. Mater. 31, 1901956 (2019).

56. Bang, S., Kim, J., Wee, D., Samsonidze, G. \& Kozinsky, B. Estimation of electron-phonon coupling via moving least squares averaging: a method for fast-screening potential thermoelectric materials. Mater. Today Phys. 6, 22-30 (2018).

57. Fröhlich, H. Electrons in lattice fields. Adv. Phys. 3, 325-361 (1954).

58. Cheng, L. \& Liu, Y. What limits the intrinsic mobility of electrons and holes in two dimensional metal dichalcogenides? J. Am. Chem. Soc. 140, 17895-17900 (2018).

59. Gonze, X. \& Lee, C. Dynamical matrices, Born effective charges, dielectric permittivity tensors, and interatomic force constants from density-functional perturbation theory. Phys. Rev. B 55, 10355-10368 (1997).

60. Canali, C., Jacoboni, C., Nava, F., Ottaviani, G. \& Alberigi-Quaranta, A. Electron drift velocity in silicon. Phys. Rev. B 12, 2265-2284 (1975).

61. Logan, R. A. \& Peters, A. J. Impurity effects upon mobility in silicon. J. Appl. Phys. 31, 122-124 (1960).

62. Morin, F. J., Geballe, T. H. \& Herring, C. Temperature dependence of the piezoresistance of high-purity silicon and germanium. Phys. Rev. 105, 525-539 (1957).

63. Norton, P., Braggins, T. \& Levinstein, H. Impurity and lattice scattering parameters as determined from Hall and mobility analysis in n-type silicon. Phys. Rev. B 8, 5632-5653 (1973).

64. Fiorentini, M. \& Bonini, N. Thermoelectric coefficients of n-doped silicon from first principles via the solution of the Boltzmann transport equation. Phys. Rev. B 94, 085204 (2016).

65. Ottaviani, G., Reggiani, L., Canali, C., Nava, F. \& Alberigi-Quaranta, A. Hole drift velocity in silicon. Phys. Rev. B 12, 3318-3329 (1975).

66. Dexter, R. N., Lax, B., Kip, A. F. \& Dresselhaus, G. Effective masses of electrons in silicon. Phys. Rev. 96, 222-223 (1954)

67. Jacoboni, C., Canali, C., Ottaviani, G. \& Alberigi-Quaranta, A. A review of some charge transport properties of silicon. Solid State Electron. 20, 77-89 (1977).

68. Mousty, F., Ostoja, P. \& Passari, L. Relationship between resistivity and phosphorus concentration in silicon. J. Appl. Phys. 45, 4576-4580 (1974).

69. Irvin, J. C. Resistivity of bulk silicon and of diffused layers in silicon. Bell Syst. Tech. J. 41, 387-410 (1962).
70. Souza, I., Marzari, N. \& Vanderbilt, D. Maximally localized Wannier functions for entangled energy bands. Phys. Rev. B 65, 035109 (2001).

71. Marzari, N., Mostofi, A. A., Yates, J. R., Souza, I. \& Vanderbilt, D. Maximally localized Wannier functions: Theory and applications. Rev. Mod. Phys. 84, 1419-1475 (2012).

72. Hicks, H. G. B. \& Manley, D. F. High purity GaAs by liquid phase epitaxy. Solid State Commun. 7, 1463-1465 (1969).

73. Rode, D. L. Electron mobility in direct-gap polar semiconductors. Phys. Rev. B 2, 1012-1024 (1970).

74. Blood, P. Electrical properties of n-type epitaxial GaAs at high temperatures. Phys. Rev. B 6, 2257-2261 (1972).

75. Nichols, K. H., Yee, C. M. L. \& Wolfe, C. M. High-temperature carrier transport in ntype epitaxial GaAs. Solid State Electron. 23, 109-116 (1980).

76. Stillman, G. E., Wolfe, C. M. \& Dimmock, J. O. Hall coefficient factor for polar mode scattering in n-type GaAs. J. Phys. Chem. Solids 31, 1199-1204 (1970).

77. Santos, R., Aminorroaya Yamini, S. \& Dou, S. X. Recent progress in magnesium-based thermoelectric materials. J. Mater. Chem. A 6, 3328-3341 (2018).

78. Tani, J. \& Kido, H. Thermoelectric properties of Bi-doped $\mathrm{Mg}_{2} \mathrm{Si}$ semiconductors. Phys. B: Condens. Matter 364, 218-224 (2005).

79. Yin, K. et al. Optimization of the electronic band structure and the lattice thermal conductivity of solid solutions according to simple calculations: a canonical example of the $\mathrm{Mg}_{2} \mathrm{Si}_{1-\mathrm{x}-\mathrm{y}} \mathrm{Ge}_{\mathrm{x}} \mathrm{Sn}_{\mathrm{y}}$ ternary solid solution. Chem. Mater. 28, 5538-5548 (2016).

80. Morris, R. G., Redin, R. D. \& Danielson, G. C. Semiconducting properties of $\mathrm{Mg}_{2} \mathrm{Si}$ single crystals. Phys. Rev. 109, 1909-1915 (1958).

81. Tran, F. \& Blaha, P. Accurate band gaps of semiconductors and insulators with a semilocal exchange-correlation potential. Phys. Rev. Lett. 102, 226401 (2009).

82. Pulikkotil, J. J. et al. Doping and temperature dependence of thermoelectric properties in $\mathrm{Mg}_{2}(\mathrm{Si}, \mathrm{Sn})$. Phys. Rev. B 86, 155204 (2012).

83. Akasaka, M. et al. The thermoelectric properties of bulk crystalline $\mathrm{n}$ - and $\mathrm{p}$-type $\mathrm{Mg}_{2} \mathrm{Si}$ prepared by the vertical Bridgman method. J. Appl. Phys. 104, 013703 (2008).

84. Zhu, T., Fu, C., Xie, H., Liu, Y. \& Zhao, X. High efficiency half-Heusler thermoelectric materials for energy harvesting. Adv. Energy Mater. 5, 1500588 (2015).

85. Zhu, T. et al. Compromise and synergy in high-efficiency thermoelectric materials. Adv. Mater. 29, 1605884 (2017).

86. Yang, J. et al. Evaluation of half-Heusler compounds as thermoelectric materials based on the calculated electrical transport properties. Adv. Funct. Mater. 18, 2880-2888 (2008)

87. He, R. et al. Achieving high power factor and output power density in p-type half-Heuslers $\mathrm{Nb}_{1-\mathrm{x}} \mathrm{Ti}_{\mathrm{x}} \mathrm{FeSb}$. Proc. Natl. Acad. Sci. U.S.A. 113, 13576-13581 (2016).

88. Hoppe, R., Lidecke, W. \& Frorath, F.-C. Zur Kenntnis von NalnS 2 und NalnSe 2 . Z. Anorg. Allg. Chem. 309, 49-54 (1961).

89. Voneshen, D. J. et al. Suppression of thermal conductivity by rattling modes in thermoelectric sodium cobaltate. Nat. Mater. 12, 1028-1032 (2013).

90. Delmas, C., Fouassier, C. \& Hagenmuller, P. Structural classification and properties of the layered oxides. Physica B+C 99, 81-85 (1980).

91. Petretto, G., Gonze, X., Hautier, G. \& Rignanese, G. M. Convergence and pitfalls of density functional perturbation theory phonons calculations from a high-throughput perspective. Comput. Mater. Sci. 144, 331-337 (2018).

92. Petretto, G. et al. High-throughput density-functional perturbation theory phonons for inorganic materials. Sci. Data 5, 180065 (2018).

93. Mahan, G. D. \& Sofo, J. O. The best thermoelectric. Proc. Natl. Acad. Sci. U.S.A. 93 , 7436-7439 (1996).

94. Baroni, S., De Gironcoli, S., Dal Corso, A. \& Giannozzi, P. Phonons and related crystal properties from density-functional perturbation theory. Rev. Mod. Phys. 73, 515-562 (2001).

95./CitationNumber>Perdew, J. P. \& Zunger, A. Self-interaction correction to densityfunctional approximations for many-electron systems. Phys. Rev. B 23, 5048-5079 (1981).

96. Perdew, J. P., Burke, K. \& Ernzerhof, M. Generalized gradient approximation made simple. Phys. Rev. Lett. 77, 3865-3868 (1996).

97. van Setten, M. J. et al. The PseudoDojo: training and grading a 85 element optimized norm-conserving pseudopotential table. Comput. Phys. Commun. 226, 39-54 (2018).

98. Troullier, N. \& Martins, J. L. Efficient pseudopotentials for plane-wave calculations. Phys. Rev. B 43, 1993-2006 (1991). 


\section{ACKNOWLEDGEMENTS}

This work is supported by Agency for Science, Technology and Research ( $A^{*}$ STAR) of Singapore (1527200024). Computational resources are provided by the National Supercomputing Centre Singapore (NSCC) and A*STAR Computational Resource Centre $\left(A^{*} C R C\right)$. K.H. also acknowledges funding from the Accelerated Materials Development for Manufacturing Program at $A^{*} S T A R$ via the AME Programmatic Fund by the Agency for Science, Technology and Research under Grant No. A1898b0043.

\section{AUTHOR CONTRIBUTIONS}

T.D. developed the computational method and performed the calculations. G.W. and S-W.Y. designed and coordinated this study. All authors provided discussions and revisions, and commented on the manuscript.

\section{COMPETING INTERESTS}

The authors declare no competing interests.

\section{ADDITIONAL INFORMATION}

Supplementary information is available for this paper at https://doi.org/10.1038/ s41524-020-0316-7.
Correspondence and requests for materials should be addressed to G.W. or S.-W.Y

Reprints and permission information is available at http://www.nature.com/ reprints

Publisher's note Springer Nature remains neutral with regard to jurisdictional claims in published maps and institutional affiliations. Attribution 4.0 International License, which permits use, sharing, adaptation, distribution and reproduction in any medium or format, as long as you give appropriate credit to the original author(s) and the source, provide a link to the Creative Commons license, and indicate if changes were made. The images or other third party material in this article are included in the article's Creative Commons license, unless indicated otherwise in a credit line to the material. If material is not included in the article's Creative Commons license and your intended use is not permitted by statutory regulation or exceeds the permitted use, you will need to obtain permission directly from the copyright holder. To view a copy of this license, visit http://creativecommons. org/licenses/by/4.0/.

(c) The Author(s) 2020 